\title{
A Stable Cell Membrane-Based Coating with Antibiofouling and Macrophage Immunoregulatory Properties for Implants at the Macroscopic Level
}

Zhiyun Dong ${ }^{\dagger}$, Xiang Ke ${ }^{\dagger}$, Shuxian Tang ${ }^{\dagger}$, Shuai $W u^{\perp}$, Wei $W u^{\perp}$, Xingyu Chen ${ }^{\ddagger}$, Jiaojiao Yang $^{\S}, J_{i n g} X i e^{\dagger}$, Jun Luo* ${ }^{, \dagger}$, Jianshu Li*,, , , ,

\section{AUTHOR ADDRESS}

${ }^{\dagger}$ College of Polymer Science and Engineering, State Key Laboratory of Polymer Materials Engineering, Sichuan University, Chengdu 610065, China

${ }^{\S}$ State Key Laboratory of Oral Diseases, West China Hospital of Stomatology, Sichuan University, Chengdu 610041, China

"Med-X Center for Materials, Sichuan University, Chengdu 610041, China

${ }^{\perp}$ Key Laboratory for Biorheological Science and Technology of Ministry of Education, State and Local Joint Engineering Laboratory for Vascular Implants, Bioengineering College of Chongqing University, Chongqing 400030, China

College of Medicine, Southwest Jiaotong University, Chengdu 610003, China Correspondence: Jun Luo (luojuncd@scu.edu.cn) and Jianshu Li (jianshu_li@,scu.edu.cn) 

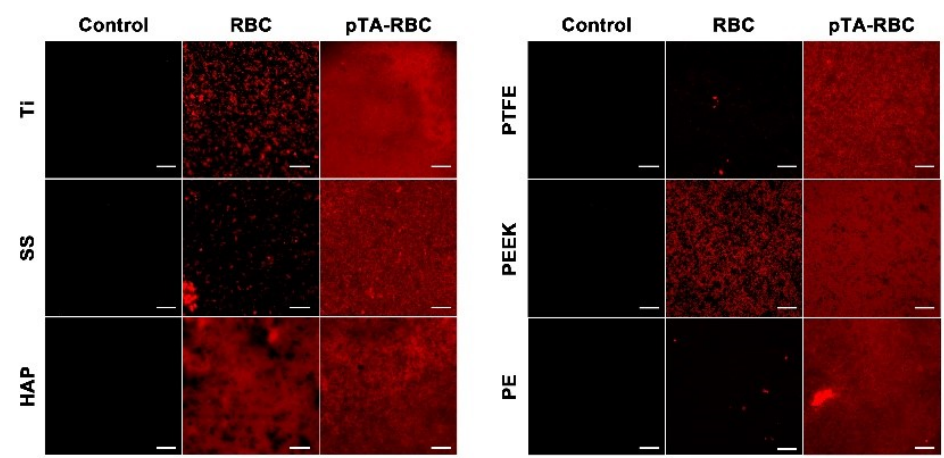

Figure S1. Fluorescence images of RBC membrane-based coating on diverse substrates.

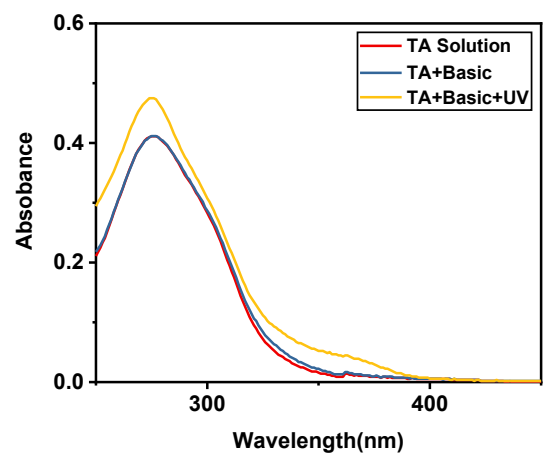

Figure S2. UV-vis spectra of TA solution, TA solution in basic buffer, and TA solution in basic buffer under UV.
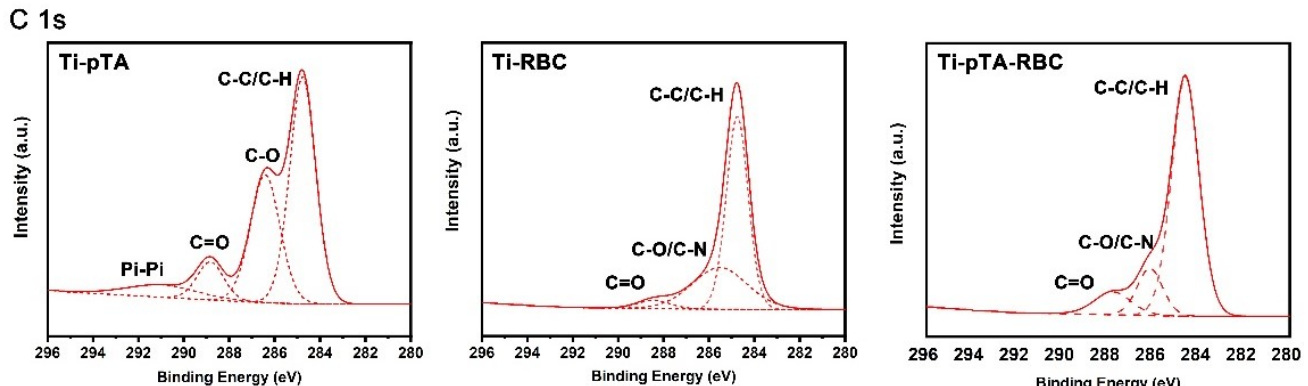

Figure S3. The high resolution of C 1s spectra for group Ti-pTA, Ti-RBC, and Ti-pTA-RBC. 
Table S1. Element content of four groups

\begin{tabular}{lcccc}
\hline & $\mathrm{C}[\%]$ & $\mathrm{O}[\%]$ & $\mathrm{N}[\%]$ & $\mathrm{P}[\%]$ \\
\hline Ti & 39.76 & 39.34 & 3.27 & 0 \\
Ti-pTA & 63.28 & 34.92 & 1.27 & 0 \\
Ti-RBC & 87.08 & 8.79 & 3.44 & 0.52 \\
Ti-pTA-RBC & 89.52 & 9.03 & 2.72 & 1.23 \\
\hline
\end{tabular}

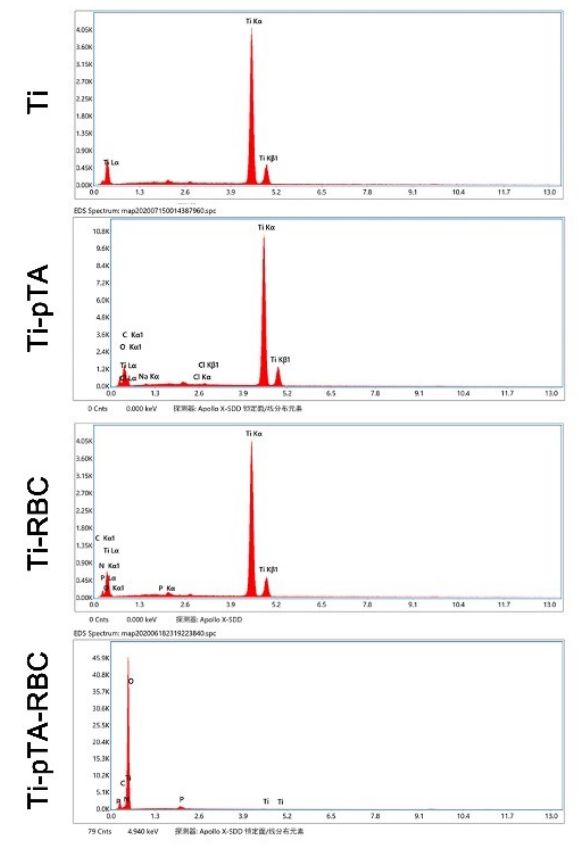

Figure S4. The EDS of four groups. 


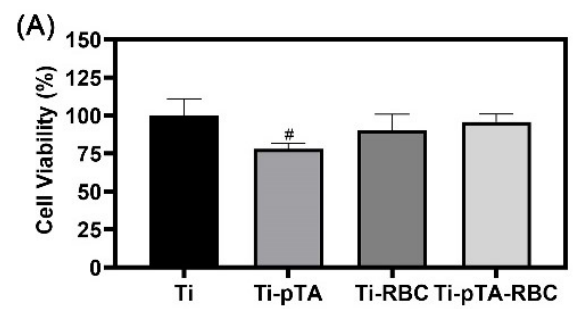

(C)
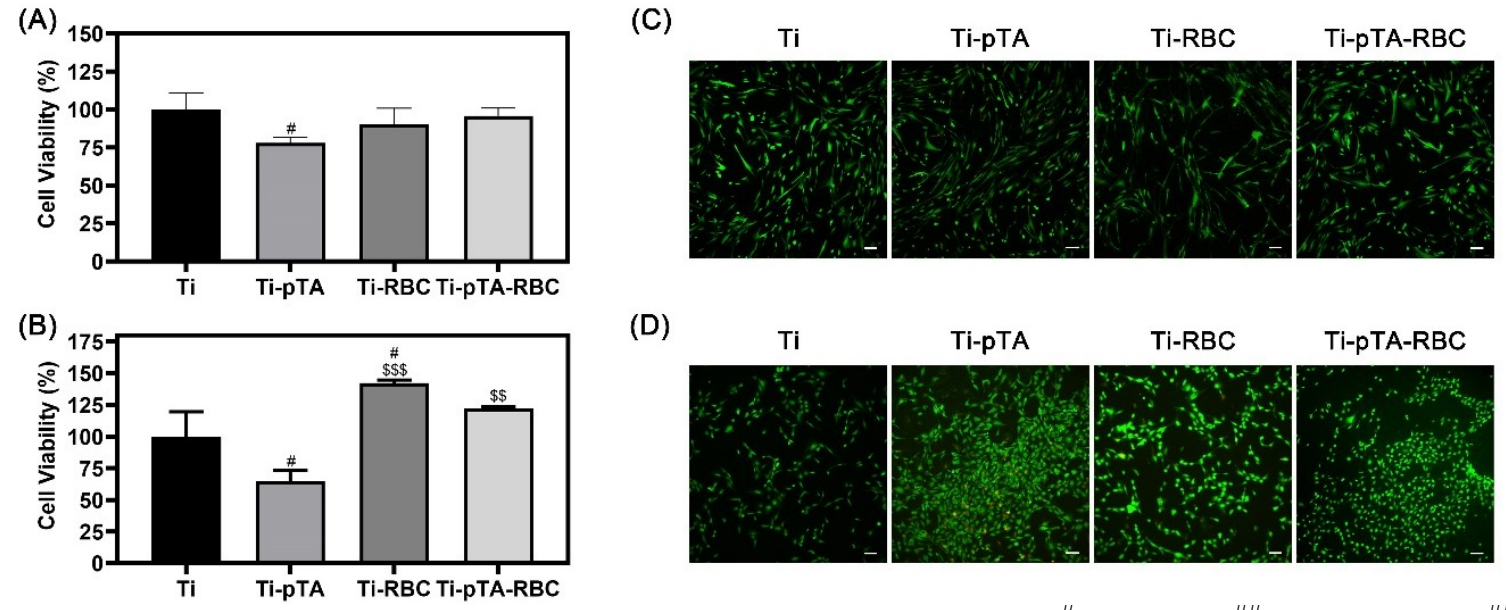

(D)

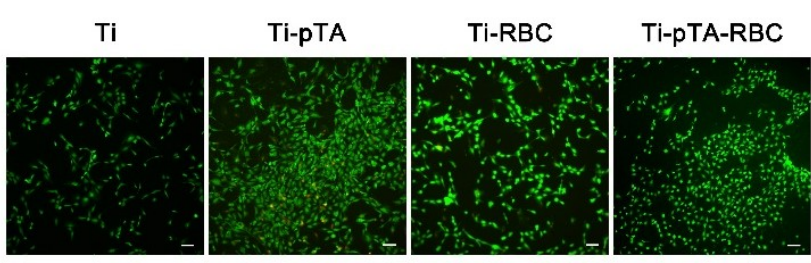

Figure S5 Cell viability of (A) HGF cells and (B) MC3T3 cells $\left({ }^{\#} p<0.05,{ }^{\# \#} p<0.01\right.$, and ${ }^{\# \# \#} p$ $<0.001$ versus the Ti group, ${ }^{\$} p<0.05,{ }^{\$} p<0.01$, and ${ }^{\$ \$} p<0.001$ versus the Ti-pTA group). The live/dead stain fluorescence images of (C) HGF cells and (D) MC3T3 cells (Scale bar: $100 \mu \mathrm{m})$.

Table S2. the primer sequences of GAPDH, IL-10, TNF- $\alpha$, CD206, and CD86 Primer sequences $\left(5^{\prime}-3^{\prime}\right)$

\begin{tabular}{lc}
\hline IL-10 Forward & ATTTGAATTCCCTGGGTGAGAAG \\
IL-10 Reverse & CACAGGGGAGAAATCGATGACA \\
TNF- $\alpha$ Forward & TCTTCTCATTCCTGCTTGTGG \\
TNF- $\alpha$ Reverse & GGTCTGGGCCATAGAACTGA \\
CD206 Forward & TTGGACGGATAGATGGAGGG \\
CD206 Reverse & CCAGGCAGTTGAGGAGGTTC \\
CD86 Forward & TCTGCCGTGCCCATTTACAA \\
CD86 Reverse & TGTGCCCAAATAGTGCTCGT \\
GAPDH Foward & ACCCAGAAGACTGTGGATGG \\
GAPDH Reverse & CACATTGGGGGTAGGAACAC
\end{tabular}

